Document downloaded from:

http://hdl.handle.net/10251/94602

This paper must be cited as:

Acosta-Davila, SC.; Chiralt A.; Santamarina Siurana, MP.; Rosello Caselles, J.; González Martínez, MC.; Cháfer Nácher, MT. (2016). Antifungal films based on starch-gelatin blend, containing essential oils. Food Hydrocolloids. 61:233-240.

doi:10.1016/j.foodhyd.2016.05.008

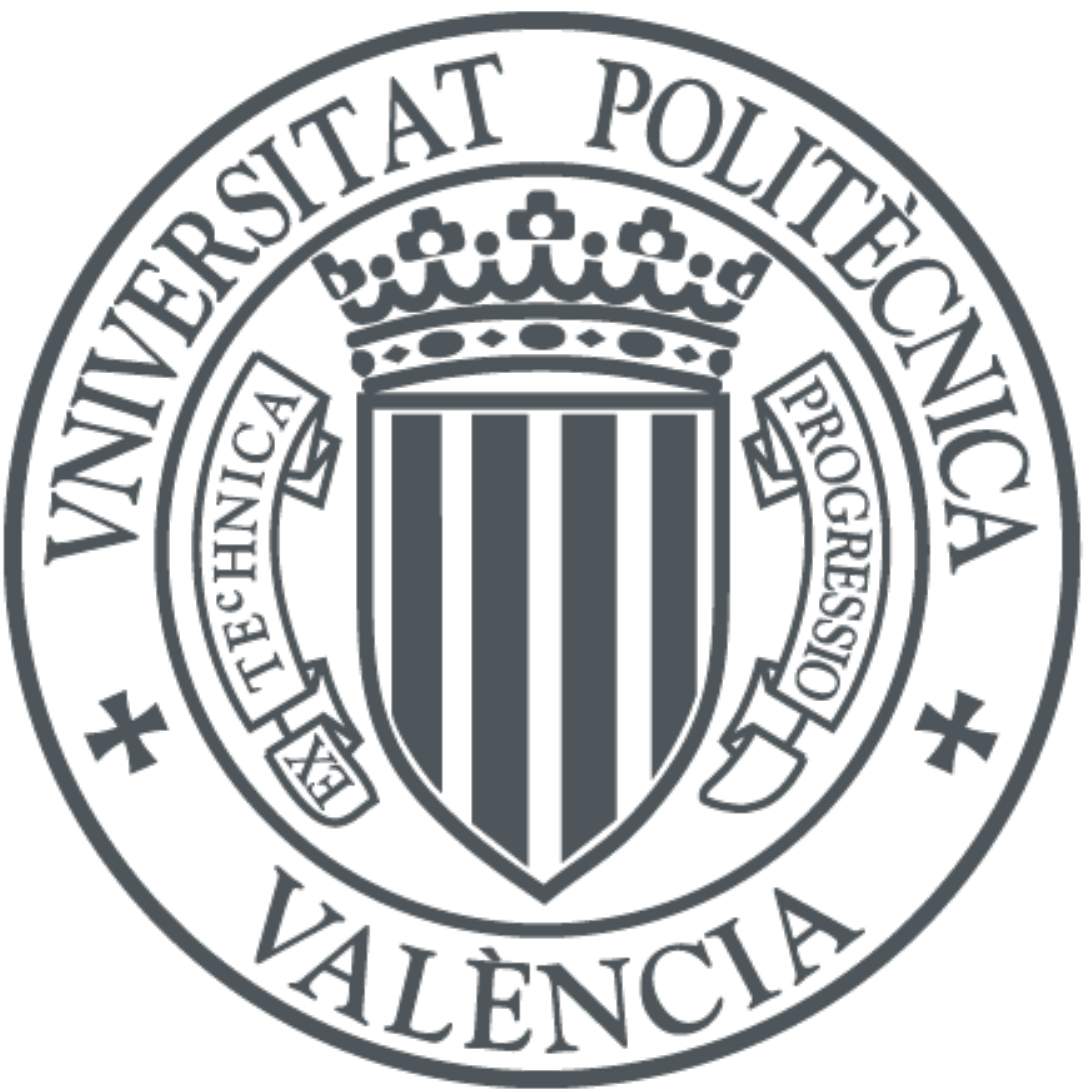

The final publication is available at

https://doi.org/10.1016/j.foodhyd.2016.05.008

Copyright Elsevier

Additional Information 


\section{Antifungal films based on starch-gelatin blend, containing essential}

2

3 Sandra Acosta, Amparo Chiralt, Pilar Santamarina, Josefa Rosello, Chelo González-

Martínez* \& Maite Cháfer.

6 Instituto de Ingeniería de Alimentos para el Desarrollo, Universitat Politècnica de València, Camino de

8 *Corresponding author: Chelo González-Martínez. Tel.: +34 3877000x83613/+34 665125730; fax: +34

5 7 Vera s/n, 46022 Valencia, Spain.

9 963877369. E-mail address: cgonza@tal.upv.es

oils.

10 


\section{Abstract}

13 The antimicrobial activity of essential oils (EO) is very well-known and it has been 14 reported that incorporating them into edible films based on biopolymers extends the 15 food's shelf-life. In this study, cinnamon, clove and oregano EO, at 25\% with respect to 16 the polymer, were incorporated into glycerol plasticized starch-gelatin blend films (ratio 17 1:1) in order to elucidate their effect on the physical (barrier, mechanical and optical), 18 structural and antifungal properties of the films. Whereas EOs exhibited no significant 19 effect on tensile behavior in the case of casting films conditioned at 53\% relative 20 humidity and $25^{\circ} \mathrm{C}$, the EO compounds did significantly reduce the water vapor and 21 oxygen permeability of the films. Likewise, the EOs increased the films' transparency 22 but reduced their gloss. Despite the fact that about $60 \%$ of the incorporated EOs were 23 lost during the film drying step, they exhibited antifungal activity against the two tested 24 fungal species, Colletotrichum gloesporoides (CG) and Fusarium oxysporum (FOG), as 25 revealed by the in vitro agar diffusion method.

26 Keywords: Colletotrichum gloesporoides, Fusarium oxysporum, antifungal, tensile, microstructural, 27 barrier, oregano, clove, cinnamon. 


\section{Introduction}

Starch is widely available from renewable sources and has a great ability to form edible films with reasonable mechanical resistance, which can be used to coat food products in order to prevent moisture loss or to protect them from oxidation. Starch films are transparent, tasteless, odorless and have very good oxygen barrier properties. Nevertheless, some drawbacks must be improved, such as the water sensitivity and retrogradation phenomena, both giving rise to changes in the film barrier and mechanical properties during storage (Cano et al., 2014; Jiménez et al., 2012; Jiménez et al., 2013). Blending starch with other compatible (such as chitosan and caseinate) or non-compatible polymers (such as poly-caprolactona) is a commonly-used strategy by which to improve the properties and stability of starch-based films (Averous \& Boquillon, 2004).

Starch-gelatin blend films have been studied by different authors (Al-Hassan \& Norziah, 2012; Fakhouri et al., 2013) who report that these blends offer advantages in terms of oxygen and water vapor barrier properties, mechanical behavior and optical parameters (Wang et al., 2003). Even though gelatin may improve the performance of starch-based materials, both polymers are highly hydrophilic, which limits the water vapor barrier capacity of the blend films.

Recently, Acosta et al. 2015, have reported the benefits of the addition of lipids on the starch-gelatin blend films with different polymer ratios. They report that cassava starch films with $50 \%$ gelatin exhibited highly adequate properties for the purposes of food coating or packaging. Gelatin blending gave rise to more resistant, harder and more extensible films than pure starch material. In this blend, although lipid incorporation improved the film's stretchability and water barrier capacity, it enhanced oxygen permeability. In this sense, essential oils (EOs) are interesting lipids that can be used in 
starch-gelatin blend films, due to their antimicrobial and antioxidant capacity which adds functionality to the film materials. Likewise, some essential oils have been effective at reducing the oxygen permeability of different polymer films in line with their oxygen scavenging activity (Bonilla et al. 2013).

The antibacterial activity of EOs has been reported by many authors (Burt, 2004; AlvesSilva et al., 2013), but few studies analyse the antifungal effect of these compounds (Avila-Sosa et al., 2012 Sánchez-González et al. 2010; $\underline{\text { Saggiorato et al., 2012; Avila- }}$ Sosa et al., 2012, Perdones et al., 2012 and 2014; Alves Silva et al., 2013; Roselló et al., 2015). The EOs affect the microbial cells via different mechanisms, including interactions with the phospholipid bilayer of the cell membrane, the disruption of enzyme systems and compromising the genetic material of bacteria (Burt et al., 2007). The use of EOs as food preservatives is limited by their strong flavour, but embedding them into a polymer matrix represents an alternative means of reducing their sensory impact while the diffusion of the antimicrobials to the product may be modulated (RuizNavajas et al., 2013). Nevertheless, the non-polar nature of the EO components requires the homogenization techniques in order to incorporate them into the aqueous film forming dispersions (FFD) of hydrocolloids, such as starch or gelatin. During the drying of the FFD cast to obtain the films, a part of the EO is lost as the water evaporates , the amount depending on the stability of the initial emulsion and the extension of the coalescence and creaming phenomena that occur in this step (Sánchez-González et al. 2011b; Perdones, et al., 2014), which affects the final bioactivity of the film. Likewise, the presence of a lipid dispersed phase and the potential interactions of lipid molecules with the polymer also affect the film’s functional properties (Chiralt et al. 2015).

The aim of this study was to analyze the effect of three different essential oils (cinnamon bark, clove and oregano) on the functional properties of cassava starch- 
bovine gelatin (ratio 1:1) blend films, in terms of the tensile, barrier, optical and structural properties, as well as their antifungal effect against two fruit fungal pathogens (Colletotrichum gloesporoides: CG and Fusarium oxysporum f.sp. gladiolo: FOG) through in vitro tests.

\section{Materials and methods}

\subsection{Raw materials}

Cassava starch, with 9.28 wt. \% amylose and an amylose:amylopectin ratio of 1:9.8, was produced by Asia CO, LDT (Kalasin, Thailand) and purchased by Quimidroga SA (Barcelona, Spain). Bovin skin type A gelatin (Bloom 220-240), used in combination with starch, was supplied by Sancho de Borja, S.L. (Zaragoza, Spain), and glycerol, used as plasticizer, was provided by Panreac Química, SA (Barcelona, Spain). Essential oils (oregano, clove and cinnamon bark) were acquired from Herbes del Moli (Benimarfull, Alicante, Spain). Table 1 shows the main components of EO used in this study and their boiling point. Stock culture of Colletotrichum gloesporoides (CG), isolated from citrus trees from Valencia (Spain), was provided by the Mediterranean Agroforest Institute (Universitat Politécnica of Valencia, Spain). Fusarium oxysporum f.sp. gladiolo (FOG) CECT 2868 was supplied by the Colección Española de Cultivo Tipo (CECT, Burjassot, Spain). These were preserved frozen in Agar Potato Dextrose (PDA, Scharlab, Barcelona, Spain), then incubated at $25^{\circ} \mathrm{C}$ until sporulation, and were used after 7 days of active growth.

\subsection{Preparation of film-forming dispersions}

The film forming dispersions were prepared from a $2 \%(\mathrm{w} / \mathrm{w})$ polymer aqueous solution, using the same proportion of cassava starch (S) and gelatin (G). Every 
103 formulation contained glycerol as plasticizer (polymer:glycerol ratio of 1:0.25).

104 Furthermore, three more formulations were prepared by adding essential oils as 105 antimicrobial agents (polymer:essential oil ratio of 1:0.25). So, four formulations were 106 considered with the following codes: SG for the control starch-gelatin blend films, SG107 C, SG-Cl and SG-O, respectively for films containing cinnamon, clove, and oregano 108 essential oils.

109 Firstly, starch and gelatin dispersions were prepared separately. Starch aqueous 110 dispersions were maintained at $95^{\circ} \mathrm{C}$ for $30 \mathrm{~min}$ to induce starch gelatinization. 111 Meanwhile, gelatin was dissolved in water at $40{ }^{\circ} \mathrm{C}$. Then, the glycerol was added to the 112 dispersion blend and homogenized by using a rotor-stator homogenizer (Ultraturrax 113 D125, Janke and Kunkel, Germany) at 13,500 rpm for $1 \mathrm{~min}$ and 20,500 rpm for 3 min 114 at $95{ }^{\circ} \mathrm{C}$ under vacuum, in agreement with previous studies (Jiménez et al., 2012). For 115 formulations containing essential oils, these were added prior to the homogenization 116 step.

117 Controlled volumes of film-forming aqueous dispersions (equivalent to $1.5 \mathrm{~g}$ of solids) 118 were cast into leveled Teflon ${ }^{\circledR}$ casting plates $\left(15 \mathrm{~cm}\right.$ diameter) and dried at $25^{\circ} \mathrm{C}$ and $11945 \% \mathrm{RH}$ for $48 \mathrm{~h}$. Then, they were peeled intact from the plates and conditioned at 53\% $120 \mathrm{RH}$ and $25^{\circ} \mathrm{C}$ in a chamber with magnesium nitrate-6-hydrate saturated solution 121 (Panreac Química, S.A., Barcelona, Spain). 
125 The microstructural analysis of the films was carried out by scanning electron

126 microscopy (SEM), using a JEOL microscope model JSM-5410 (Japan). Prior to

127 testing, films were equilibrated in desiccators with $\mathrm{P}_{2} \mathrm{O}_{5}$ in order to remove any water

128 present in the samples. SEM observations were carried out by considering the surfaces

129 and cross sections of film samples. Films were frozen in liquid nitrogen, and then

130 cryofractured to observe the cross section. Samples were fixed on copper stubs, gold 131 coated to make them conductive (for 1.5 minutes) and observed directly with an 132 accelerating voltage of $10 \mathrm{KV}$.

\section{2.3.2. Water vapor permeability}

134 The water vapor permeability (WVP) of films was determined following the gravimetric method ASTM E96-95 (1995) for a 53-100\% relative humidity gradient at $25^{\circ} \mathrm{C}$ by using Payne permeability cups (Payne, Elcometer SPRL, Hermelle/s Argenteau, Belgium) of $3.5 \mathrm{~cm}$ diameter. The RH gradient of 53-100\% of the cabinet was obtained using oversaturated solutions of $\mathrm{Mg}\left(\mathrm{NO}_{3}\right)_{2}$ and distilled water, respectively. The weight of the cup was measured every $2 \mathrm{~h}$ using an analytical balance ( $\pm 0.00001 \mathrm{~g})$. The water vapor transmission (WVTR) was determined from the slope obtained from the regression analysis of weight loss data versus time, once the steady state had been reached, divided by the film area. Water vapor permeability (WVP) values were obtained, in quadruplicate, according to previous studies (Jiménez et al. 2012).

145 The oxygen permeability (OP) of the films was analyzed in film samples $\left(50 \mathrm{~cm}^{2}\right)$ by using an Oxtran system (Mocon, Minneapolis, USA), following the standard method 147 (ASTM D3985-05, 2002) at 53\% RH and $25^{\circ} \mathrm{C}$. Films were exposed to pure nitrogen flow on one side and pure oxygen flow on the other side. OP was calculated by dividing 
the oxygen transmission rate by the difference in oxygen partial pressure between the two sides of the film, and multiplying by the average film thickness. At least two replicates per formulation were taken.

\subsubsection{Tensile behavior}

153 The tensile properties were measured using a universal test machine (TA.XT plus

154 model, Stable Micro Systems, Haslemere, England) and following the ASTM standard method D882 (ASTM, 2001). Equilibrated samples (8 per formulation) were mounted in the film-extension grips (model A/TG, Stable Micro System, Haslemere, England) of the testing machine and stretched at a rate of $50 \mathrm{~mm} \cdot \mathrm{min}^{-1}$ until breaking. The relative humidity of the environment was held at nearly 53\% during the tests, which were performed at $25^{\circ} \mathrm{C}$.

The force-distance data obtained in the test were transformed into true stress-Henky strain curves, which allow the mechanical parameters to be calculated: elastic modulus (EM), tensile strength at break (TS) and percentage of elongation at break (E).

\subsubsection{Optical properties}

The transparency of the films was determined by applying the Kubelka-Munk theory (Hutchings, 1999) for multiple scattering to the reflection spectra. The surface reflectance spectra of the films were determined from 400 to $700 \mathrm{~nm}$ with a spectrocolorimeter CM-3600d (Minolta Co., Tokyo, Japan) on both a white and a black background. Internal transmittance (Ti) of the films was quantified using eq 1 . In this equation, $\mathrm{R}_{0}$ is the reflectance of the film on an ideal black background. Parameters a and b were calculated by eqs 2 and 3, where $\mathrm{R}$ is the reflectance of the sample layer backed by a known reflectance Rg. Measurements were taken in triplicate for each 172 sample on the free film surface during its drying. 


$$
\mathrm{a}=\frac{1}{2} \cdot\left(\mathrm{R}+\frac{\mathrm{R}_{0}-\mathrm{R}+\mathrm{R}_{\mathrm{g}}}{\mathrm{R}_{0} \mathrm{R}_{\mathrm{g}}}\right)
$$

$$
\mathrm{b}=\left(\mathrm{a}^{2}-1\right)^{1 / 2}
$$

176 The gloss of the films was measured at a $60^{\circ}$ incidence angle, using a flat surface gloss 177 meter (Multi.Gloss 268, Minolta, Germany), according to the ASTM standard D-523 178 (ASTM, 1999). Three films of each formulation were measured over a black matte standard plate. Results were expressed as gloss units, relative to a highly polished surface of standard black glass with a gloss value close to 100 .

Antifungal properties of films against FOG and CG were determined by the agar diffusion method using film discs of $2.4 \mathrm{~cm}$ diameter, which were placed on the plate with the fungus spores. To this end, for each fungus, a suspension of $5 \times 10^{6} \mathrm{CFU} / \mathrm{mL}$ spores in water with Tween $80(0.1 \%)$ was prepared from a colony of 7 days' active growth in PDA medium. This suspension was distributed in plates and left to solidify. Then, the film discs were deposited on the different plates and they were incubated under the optimal conditions for each fungal species. The diameter of the growth inhibition halo was measured at 48 and 72 hours in all cases. Six replicates per treatment and control sample (without film) were made.

\subsubsection{Statistical analysis}

The analysis of data was performed through the analysis of variance (ANOVA) using the Statgraphics Plus 5.1. software (ManugisticsCorp., Rockville, MD). Fisher's least significant difference (LSD) procedure was used at the 95\% confidence level. 


\section{Results and discussion}

3.1. Film microstructure and losses of essential oils during film formation.

The microstructure of films, which depends on the interactions between film components and drying conditions, seriously affects the physical properties of the final materials, such as barrier, mechanical and optical properties. In films containing lipids, such as essential oils, the final structure is also affected by the structural characteristics and stability of the film-forming emulsion. The different emulsion destabilization mechanisms (flocculation, creaming and coalescence) that occur during the film drying step determine the final distribution and size of lipid droplets in the matrix (Villalobos, et al. 2005; Fabra et al. 2009).

207

Figure 1 shows the SEM micrographs corresponding to the surfaces and cross-sections of the studied films. SEM observations show that starch-gelatin composite films exhibited heterogeneous structures and irregularities in both surface and cross-section images. Round formations are observed on the surface of these films, while in film cross-sections, fibrous regions appear. These formations have been related with the lack of total miscibility of starch and gelatin and the polymer phase separation, giving rise to a starch rich phase interpenetrated with a gelatin rich phase (Acosta et al. 2015). In the gelatin phase, helical chain conformation and association (triple helix) occur in a similar way to in the original collagen structure, as observed by Hassan et al. (2009) and Al Hassan and Norziah, (2012) in gels of starch-gelatin. Gelatin association domains appear as fibrous zones differentiated in the amorphous starch matrix. On the surface of the film, the rounded formations (droplets in non-dried film) reflect the migration of gelatin-rich liquid phase to the film surface during the film drying step due to the phase 
separation of polymer solution and the lower density of the protein phase with respect to the starch-rich solution (Acosta et al. 2015).

222 The addition of EOs also promoted irregularities on the film surface due to the low 223 miscibility of these lipid compounds in the polymer matrix. EOs are dispersed in the 224 film-forming formulations and, during the film drying process, flocculation, coalescence and creaming phenomena occur, leading part of the oil droplets to the film surface, where the EO evaporates. The footprints of oil droplets are clearly appreciated in the film containing CL oil, whereas wider traces can be seen for films with $\mathrm{O}$ essential oil. Oil could be evaporated during the film drying and conditioning steps or under the microscopic observation at high vacuum. However, for cinnamon oil, although the lipid footprint at the surface level is not evident, a different surface topography can be observed, as compared with control films without essential oils, which suggests the presence of oil on the surface at some time during the film preparation. The cross-sections of films containing EOs also reveal the presence of numerous holes that correspond to the location of oil droplets. These could evaporate partially on the cryo-fractured surface during SEM observations due to the high vacuum. They appeared elongated in shape, which can be justified by their deformation produced during the film drying and subsequent packing of the polymer chains, as has been previously described (Sánchez-González et al., 2010; Bonilla et al. 2013). Films with O essential oil exhibit a porous structure, but without well differentiated drops, which suggests their coalescence during film drying and the formation of essential oil channels that become entrapped in the polymer matrix. The proportion of porous zones 242 is higher near the free surface of the film during drying, in agreement with the oil 243 creaming to the top of the film. These results highlight a greater instability of oregano oil emulsion during film drying. 
The appearance of films with essential oils is similar to that previously described for hydrocolloid matrices with essential oils (Perdones et al., 2012). Differences in the distribution of essential oils in the polymer matrix are related with the different composition, volatility and affinity (interactions) between them and the polymers of matrix, which, in turn, affect the stability of the initial emulsion and its development during the film drying step. Notable differences in the structure and distribution of oil droplets in the film matrix have previously been observed for gelatin films with essential oils (Ahmad et al., 2012), starch films with fatty acids (Jiménez et al., 2012) or chitosan and HPMC films with essential oils (Sánchez-González et al., 2010). In this sense, the quantification of the total EO present in the film after the drying step is relevant, not only because of its effect on the functional film properties, such as mechanical or barrier performance, but also due to its active properties, such as antimicrobial or antioxidant activity.

The mean percentages of EO lost during the film preparation processes are estimated for the obtained films. This was carried out from the difference between the weights of the dried film (without moisture) and the total solids of the cast film-forming dispersions with respect to the corresponding initial amount of EO. The losses of EO were about 56-59 \% for C and CL, whereas they were significantly higher (66\%) for the O EO. This could be due to the greater instability of the initial emulsion of this EO, as deduced from the SEM micrographs of the films, which leads to the coalescence and creaming of the oil droplets, which evaporate at the film's surface during the drying step. The greater volatility of the main compounds of oregano EO (50\% carvacrol and $21 \%$ thymol) in comparison with cinnamon (60\% eugenol) and clove (90\% eugenol) (Table 1) could also enhance the oil compounds lost. During the film's drying period, water and EO compounds evaporate together at a lower temperature than pure water 
270 (immiscible blends) from the film surface until a certain level of concentration of the

271 film solids was reached, when the oil droplets remain entrapped in the highly viscous

272 aqueous system and do not cream to the surface. From this point, the oil droplets

273 become encapsulated in the film matrix and the release of the oil components from the

274 dried films due to evaporation takes place very slowly, as deduced by their very small

275 weight loss throughout time.

\section{3.2. Barrier properties}

277 In Table 2, the average values of water vapor and oxygen permeabilities of starchgelatin based films are also shown. The addition of EO to starch-gelatin films caused a decrease in the water vapor permeability, as can be expected from the increase in their hydrophobicity and the interruptions in the matrix continuity for mass transfer. Water molecules should diffuse mainly through the continuous polymeric phase, where the presence of lipid droplets represents discontinuities that cause an effective increase in the tortuosity factor for water transfer in the matrix. No significant differences between the WVP values of films with different EOs were obtained.

The effect of EO incorporation on the water barrier properties of the films has been analyzed in previous studies and different results have been reported. This effect depends on different structural factors, such as the kind of matrix, the composition and amount of oil added and the interactions with the matrix (Sánchez-González et al., 2011b). The process of water vapor transfer in films depends on the hydrophilichydrophobic balance in the matrix and the final film microstructure. Sánchez-González et al. (2011b) found a reduction in the WVP in chitosan and HPMC matrices when 0.5, 1 and $2 \%$ of bergamot, tea tree, lemon and bergamot EO were added. In general, the higher the EO concentration, the lower the WVP values in both matrices. The kind of 
EO significantly affected this property in HPMC matrices. The films containing citrus

295 EO exhibited a greater WVP reduction, as compared with films based on tea tree EO.

This was explained by the more hydrophobic nature of the main compounds of citrus

values to a lesser extent in the chitosan matrix due to its greater hydrophilic nature.

Kechichian et al (2010) found a reduction in the WVP in cassava starch films when cinnamon and clove powder (0,2 g/100 g of film) were added to the matrix. At higher concentrations, the WVP values increased.

Ahmad et al (2012) obtained a different effect in gelatin-based films with bergamot and lemon EO. At EO concentrations higher of over 5\%, the WVP slightly increased in the films with bergamot oil, while those containing lemon EO showed a WVP reduction. This was related with the different hydrophobic nature of these oils. Interactions between the EO compounds and the hydrophobic parts of proteins could promote an overall decrease in the hydrophobic nature of the matrix (Perez-Gago y Krochta, 2001). The oxygen permeability values (Table 2) also showed a significant decrease when EOs were added to the films, as compared to the values of the control films, but using different oils did not lead to any significant differences between the films. Lipid incorporation usually promotes higher oxygen permeability of the films due to the greater gas solubility in the lipid phase (Fabra et al., 2012). However, essential oils (from thyme and basil) and other antioxidants notably reduced the oxygen permeability of starch-chitosan blend films (Bonilla et al., 2013). The poorer oxygen solubility in these more polar oils and the presence of antioxidant compounds that can play an oxygen scavenging role are the reasons for the positive effect of EO on the oxygen barrier properties of films. 
320 The mechanical and optical properties of different films, with and without essential oils, are shown in Table 3. In general, the mechanical parameter exhibited few changes as a result of the EO incorporation. The values of elasticity modulus (EM), tensile strength (TS) and deformation at fracture point (\%E) of the control and EO-containing films are not significantly different.

Previous studies have described different effects of the incorporation of EO into polymeric matrices. Sanchez-Gonzalez et al. (2011b) found similar tendencies in films based on HPMC and chitosan after additions of different EO concentrations (0.5- 1 and 2\%). The addition of EO caused a decrease in EM, TS and \%E. The kind of EO only had a significant effect on chitosan films. Kechichian et al. (2010) also found that there was a decrease in the mechanical properties after incorporating powder of cinnamon and clove into starch films. However, Bonilla et al. (2013) found an increase in the film's rigidity and tensile strength in starch-chitosan blend films with basil essential oil; with thyme oil, meanwhile, the films only were slightly less extensible. In this study, authors found that in pure chitosan films, the incorporation of thyme and basil EO caused a significant decrease in the rigidity and tensile strength of the films (the higher the essential oil concentration, the more intense the effect), while the films gained extensibility.

All of the described results highlight that the effect of EO on the film's mechanical properties depends on the type and concentration of EO, the kind of polymer matrix and the specific interactions between components (Ahmad et al. 2012), which determine the effective adhesion forces at the polymer-oil interphase. In general, if essential oils, or other immiscible, non-polymeric components, are added in excessive concentrations, the films support a great quantity of dispersed phase and the structure becomes too 
heterogeneous, with a great number of discontinuities, which imparts a highly fragile nature to the matrix. Nevertheless, if the optimal level of essential oil is incorporated, the film's properties can be enhanced depending on the specific interactions between components.

The few changes obtained in the mechanical properties of starch-gelatin films as a result of the incorporation of essential oils reveal that the concentrations used are suitable for preserving the mechanical behavior of the films based on starch:gelatin, while imparting other positive effects, such as the improvement in the barrier properties commented on above and potential active properties.

Table 3 also shows the values of the optical properties of the films. Film gloss is related with surface morphology (Villalobos et al. 2005) and, in general, the smoother, less irregular the film surface, the glossier the film. The gloss values revealed that the incorporation of EO caused a reduction in this parameter in comparison with the control films. As previously commented on, EOs introduce structural irregularities at the surface level of the films, which directly reduce film gloss. The kind of EO affected the gloss of the films; the oregano EO produced the glossiest films and the cinnamon oil the least glossy. The surface topography of the films, influenced by the oil creaming during the drying period, and possible oil exudation to the surface in the dried film, will be determining factors in the film's final gloss.

The control films exhibited lower transparency values, evaluated through Ti, than those that contain EO, despite the presence of an oil dispersed phase that could contribute to light dispersion and an increase in opacity. In fact, the incorporation of EO at different concentrations in chitosan and HPMC matrices caused a decrease in the transparency of all the films (Sánchez-González et al. 2011b). Nevertheless, in starch-gelatin films, Ti values significantly increased as the EO was incorporated, regardless of the kind of oil . 
369

370

371

372

373

374

375

376

377

378

379

380

381

382

383

384

385

386

387

388

389

390

391

392

393

This could be due to the less compact nature of the polymer matrix in the presence of the EOs and the possible inhibition of starch and gelatin crystallization phenomena. In fact, Jiménez et al. (2012), observed a decrease in the transparency and gloss of corn starch films during storage attributable to the progress of amylose crystallization. The presence of oils could help to limit these crystallization phenomena, leading to more transparent films.

\subsection{Antifungal activity in vitro tests}

Table 4 shows the results of the antifungal effectiveness of the films containing EO through the diameter of inhibition of the fungal growth for Fusarium oxysporum (FOG) and Colletotrichum Gloeosporiodes (CG), after 48 and 72 hours of incubation. The control plates (without film) were used to compare the fungal growth. The results obtained in the control plates showed a growth equivalent to $2 / 3$ of the total plate surface after $24 \mathrm{~h}$, whereas at $72 \mathrm{~h}$, the entire surface was covered by the fungus.

The three films containing EOs were effective against the fungal growth. In general, this effect was somewhat more marked in films containing cinnamon essential oil against the Fusarium oxysporum (FOG) fungus, while films with clove and cinnamon essential oils were more active against the Colletotrichum Gloeosporiodes (CG) fungus. The films with oregano essential oil were always less effective than the other two. These differences may be related, among other factors, with the nature and composition of the EOs, the ratio of EO:polymer in the film and possible interactions between polymers and active compounds which can affect their diffusion in the medium (SánchezGonzález et al., 2011a). In these essential oils, eugenol, carvacrol and thymol are the main phenols. Eugenol is abundant in clove (90\%) and cinnamon (60\%), and is the main volatile compound of the oils; while in oregano oil, high percentages of carvacrol (50\%) and thymol (21\%) are usually found (Roselló, J.; Sempere, F.; Sanz-Berzosa, I.; 
Chiralt, A.; Santamarin M. P. (2015). Moreover, the antimicrobial activity of essential oils is not only attributable to their main constituents. Different studies concluded that essential oils are more effective than isolated active compounds or mixtures of these, probably due to the development of synergistic effects (Mourey and Canillac, 2002; Ranasinghe et al., 2002).

The greater effectiveness of films containing cinnamon EO is coherent with previous studies about the in vitro analysis of the antimicrobial effectiveness of films based on Arabic gum against pathogens causing antracnosis (C. musae and C. Gloeosporiodes) (Maqbool et al., 2011). Films containing cinnamon EO were also reported to exert a good rot control when applied to papaya (Acosta, 2015-TESIS)

404

405

\section{Conclusions}

406

407

408

409

410 matrix due to their lack of miscibility. Their presence in the film led to a significant reduction in the water vapor permeability of starch-gelatin films, a $17-30 \%$ reduction in oxygen permeability (depending on the essential oil) and an increase in transparency, without causing any negative effects in the film's mechanical behavior, apart from the 416 fact that it does reduce the film's gloss. Films with EOs exhibited a notable antifungal activity against the two tested fungus species; this was particularly true in the cases of 
418 those containing cinnamon EO against Fusarium oxysporum f.sp. gladiolo and those containing clove EO against Colletotrichum gloesporoides.

\section{Acknowledgements}

422

The authors acknowledge the financial support provided by the Ministerio de Economía

y Competitividad-Spanish Government (AGL2013-42989-R)

\section{References}

Acosta, S., Jiménez, A.; Cháfer, M.; González-Martínez, C.; Chiralt, A. (2015). Physical properties and stability of starch-gelatin based films as affected by the addition of esters of fatty acids. Food Hydrocolloids, 49,135-143.

Al-Hassan, A.A., \& Norziah, M.H. (2012). Starch-gelatin edible films: Water vapor permeability and mechanical properties as affected by plasticizers. Food Hydrocolloids, 26 (1), 108-117.

Ahmad M., Benjakul S., Prodpran T., Winarni Agustini T. (2012). Physico-mechanical and antimicrobial properties of gelatin film from the skin of unicorn leatherjacket incorporated with essential oils. Food Hydrocolloids, 28 (1), 189-199.

Alves-Silva, J.M., Dias dos Santos, S.M., Pintado, M.E., Pérez-Álvarez J.A., Fernández-López, ,J. , \& Viuda-Martos, M. (2013). Chemical composition and in vitro antimicrobial, antifungal and antioxidant properties of essential oils obtained from some herbs widely used in Portugal. Food Control, 32 (2), 371-378.

Averous, L., \& Boquillon, N. (2004). Biocomposites based on plasticized starch: thermal and mechanical behaviours. Carbohydrate Polymers, 56 (2), 111-122. 
Avila-Sosa, R., Palou, E., Jiménez-Munguía, M. T., Nevárez-Moorillón, G. V., Navarro-Cruz, A. R., \& López-Malo, A. (2012). Antifungal activity by vapor contact of essential oils added to amaranth, chitosan, or starch edible films. International Journal of Food Microbiology, 153(1-2), 66-72.

Bonilla, J., Talón, E., Atarés, L., Vargas, M., Chiralt, A (2013). Effect of the incorporation of antioxidants on physicochemical and antioxidant properties of wheat starch-chitosan films. Journal of Food Engineering, 118(3), 271-278.

Burt, S. (2004). Essential oils: their antibacterial properties and potential applications in foods- a review. International Journal of Food Microbiology, 94(3), 223-253.

Burt, S. A., Der Zee, R. V., Koets, A. P., De Graaff, A. M., Van Knapen, F., Gaastra, W., et al. (2007). Carvacrol induces heat shock protein 60 and inhibits synthesis of flagellin in Escherichia coli O157:H7. Applied and Environmental Microbiology, 73, 4484-4490.

Cano, A., Jiménez, A., Cháfer, M., González, C., \& Chiralt, A. (2014). Effect of amylose:amylopectin ration and rice bran addition on starch films properties. Carbohydrate Polymers, 111, 543-555.

Chiralt, A., Talens, P., Monedero, F.M., Fabra, M.J. (2015). Effect of different components of edible/biodegradable composite films on water relationships in the polymer matrix. In Gutiérrez-López, G., Alamilla-Beltran, L., Buera, M.d.P., WeltiChanes, J., Parada-Arias, E., Barbosa-Cánovas, G.V. (Eds.), Water stress in biological, chemical, pharmaceutical and food systems. (pp. 101 - 114). Springer International Publishing AG (New York).

Fabra, M.J.; Talens, P.; Chiralt, A. (2009). Microstructure and optical properties of sodium caseinate films containing oleic acid - beeswax mixtures. Food Hydrocolloids, 23(3), 676-683 
Fabra, M. J.; Talens, P.; Gavara, R.; Chiralt, A. (2012) Barrier properties of sodium caseinate films as affected by lipid composition and moisture content. Journal of Food Engineering, 109(3), 372-379.

Fakhouri, F.M., Costa, D., Yamashita, F., Martelli, S.M., Jesus, R.C., Alganer, K., Collares-Queiroz, F.P., \& Innocentini-Mei, L.H. (2013). Comparative study of processing methods for starch/gelatin films. Carbohydrate Polymers, 95(2), 681689.

Firoozmand, H., Murray B., Dickinson E. (2009). Microstructure and rheology of phase-separated gels of gelatin + oxidized starch. Food Hydrocolloids, 23(4), 10811088.

Hutchings, J. B. (1999). Food color and appearance (2nd ed.). Gaithersburg, Maryland, USA: Aspen Publishers, Inc.

Jiménez, A., Fabra, M.J., Talens, P., \& Chiralt, A. (2012). Effect of re-crystallization on tensile, optical and water vapour barrier properties of corn starch films containing fatty acids. Food Hydrocolloids, 26 (1), 302-310.

Jiménez, A., Fabra, M.J., Talens, P., \& Chiralt, A. (2013). Phase transitions in starch based films containing fatty acids. Effect on water sorption and mechanical behaviour. Food Hydrocolloids, 30 (1), 408-418.

Kechichian V., Ditchfield C., Veiga-Santos Pr., Tadini C. (2010).Natural Antimicrobial ingredients incorporated in biodegradable films. Food Science and Technology, 43, 1088-1094.

Maqbool M., Ali A., Alderson P.G., Mohamed M.T.M., Siddiqui Y., Zahid N. (2011). Postharvest application of gum Arabic and essential oils for controlling anthacnose and quality of banana and papaya during cold storage. Postharvest Biology and Technology, 62(1), 71-76. 
491

492

493

494

495

496

497

498

499

500

501

502

503

504

505

506

507

508

509

510

511

512

513

514

515

Mourey A., Canillac N. (2002). Anti-listeria monocytogenes activity of essential oils components of conifers. Food Control, 13(4), 289-292.

Perez-Gago MB., Krochta JM., (2001). Lipid particle size effect on water vapour permeability and mechanical properties of whey protein/beeswax emulsion films. Journal of Agricultural and Food Chemistry, 49 (2), 996-1002.

Perdones, A., Sánchez-González, L., Chiralt, A., \& Vargas, M. (2012). Effect of chitosan lemon essential oil coatings on storage-keeping quality of strawberry. Postharvest Biology and Technology, 70, 32-41.

Perdones, A., Vargas, M., Atarés, L., Chiralt, A. (2014) Physical, antioxidant and antimicrobial properties of chitosan-cinnamon leaf oil films as affected by oleic acid. Food Hydrocolloids, 36, 256-264.

Ranasinghe, L.; Jayawardena, B.; \& Abeywickrama, K. (2002). Fungicidal activity of essential oils of Cinnamomum zeylanicum (L.) and Syzygium aromaticum (L.) Merr et L.M. Perry against crown rot and anthracnose pathogens isolated from banana. Letters in Applied Microbiology, 35, 208-211.

Roselló, J., Semperea, F., Sanz-Berzosa, I., Chiralt, A., Santamarina, P. (2015). Antifungal activity and potential use of essential oils against Fusarium culmorum and Fusarium verticillioides. Journal of Essential Oil Bearing Plants, 18(2), 359367.

Ruiz-Navajas, Y., Viuda-Martos, M., Sendra, E., Perez-Alvarez, J.A., \& FernándezLópez, J. (2013). In vitro antibacterial and antioxidant properties of chitosan edible films incorporated with Thymus moroderi or Thymus piperella essential oils. Food Control, 30(2), 386-392.

Saggiorato, A.G., Gaio, I., Treichel, H., De Oliveira, D., Cichoski, A.J., \& Cansian, R.L. (2012). Antifungal activity of basil essential oil (Ocimum basilicum L.): 

evaluation in vitro and on an Italian-type sausage surface. Food and Bioprocess Technology, 5, 378-384.

Sanchez-González, L., Cháfer, M, Chiralt, A. and González-Martínez, C., (2010). Physical properties of chitosan films containing bergamot essential oil and their inhibitory action on Penicilium Italicum. Carbohydrate Polymers, 82, 277-283.

522

Sanchez-Gonzalez, L., Cháfer, M., González-Martínez, C., Chiralt, A., Desobry S.

523 (2011a). Study of the release of limonene present in chitosan films enriched with bergamot oil in food simulants. Journal of Food Engineering, 105(2), 138-143.

524

525

Sanchez-Gonzalez, L., González-Martínez, C., Chiralt, A. Chafer, M. (2011b) Effect of essential oils on properties of film forming emulsions and films based on hydroxypropylmethylcellulose and chitosan. Journal of Food Engineering, 105(2), 246-253.

Villalobos, R.; Hernández, P.; Chanona, J.; Gutiérrez-López, G.; Chiralt, A. (2005) Gloss and transparency of hydroxypropilmethylcellulose films containing surfactants as affected by their microstructure. Food Hydrocolloids, 19, 53-61.

Wang Y., Rakotonirainy M., Papua W., (2003) Thermal behavior of zeina-base biodegradable films. Starch/Starke, 55, 25-29.

533

534

535

536

537

538

539 


\section{$541 \quad$ FIGURE CAPTIONS}

542 Figure 1. SEM images of the surfaces (left column) and the cross-sections (right

543 column) of starch-gelatin films, containing or not different essential oils (cinnamon (C),

544 clove (CL) and (O) oregano).

545

546

547

548

549

550

551

552

553

554

555 

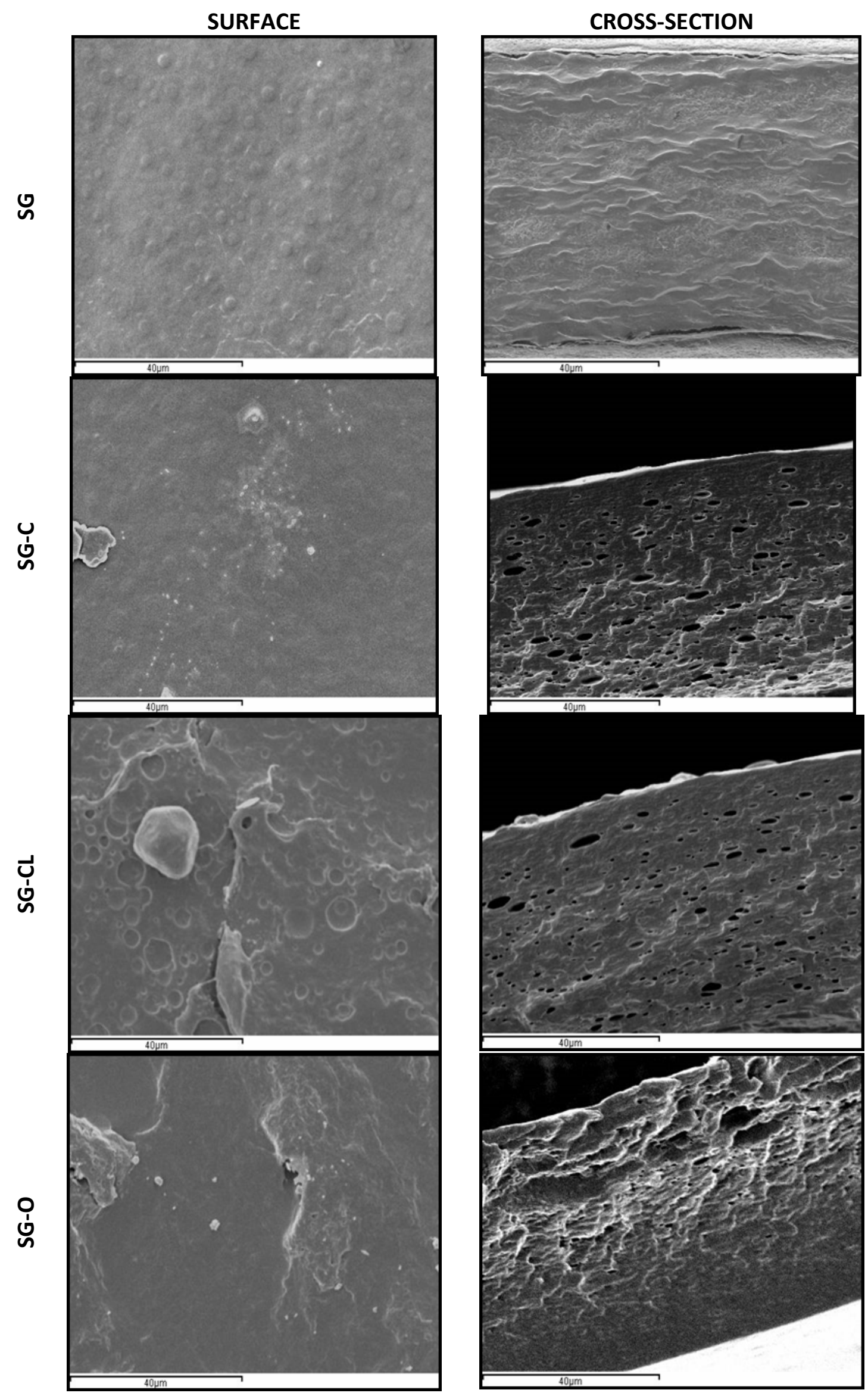
557 Table 1. Average value ${ }^{(*)}$ (wt. \%) of the main EO components and their melting point $558\left({ }^{\circ} \mathrm{C}\right)$ between brackets (adapted from Rosello, et al 2015).

\begin{tabular}{lccc}
\hline $\begin{array}{l}\text { Components } \\
\left.\text { (melting point, }{ }^{\circ} \mathrm{C}\right)\end{array}$ & Cinnamon & Clove & Oregano \\
& EO (\%) & EO (\%) & EO (\%) \\
\hline Eugenol $\left(256^{\circ} \mathrm{C}\right)$ & 60 & 90 & - \\
Acetato de cinamilo $\left(265^{\circ} \mathrm{C}\right)$ & 5 & - & - \\
Acetato de eugenilo $\left(268^{\circ} \mathrm{C}\right)$ & 18 & - & - \\
Benzoato de benzilo $\left(323^{\circ} \mathrm{C}\right)$ & 4 & - & - \\
Beta-Cariofileno $\left(280^{\circ} \mathrm{C}\right)$ & - & 7 & - \\
Timol $\left(233^{\circ} \mathrm{C}\right)$ & - & - & 21 \\
Carvacrol $\left(238^{\circ} \mathrm{C}\right)$ & - & - & 50 \\
Gamma-Terpineno $\left(183^{\circ} \mathrm{C}\right)$ & - & - & 9 \\
Para-Cimeno $\left(177^{\circ} \mathrm{C}\right)$ & - & - & 11 \\
\hline values depend on several factors as method of extraction, geographical location, genetic and \\
environmental conditions, among others,
\end{tabular}

561

562

563

564

565

566

567

568 
570 Table 2. Barrier properties (WVP: water vapour permeability and OP: oxygen 571 permeability), and loss of essential oil after films drying. C: cinnamon; Cl: clove; O: 572 oregano. Mean values, standard deviation and ANOVA results.

\begin{tabular}{lccc}
\hline FILMS & $\begin{array}{c}\text { WVP } \\
\left.\text { (g.mm/kPa.h.m }{ }^{2}\right)\end{array}$ & $\begin{array}{c}\text { OPx10 } \\
\left(\mathrm{cm}^{3} / \mathrm{m} \cdot \mathrm{sec} . P a\right)\end{array}$ & $\begin{array}{c}\text { Loss of essential } \\
\text { oil (\%) })^{(1)}\end{array}$ \\
\hline SG & $5,9 \pm 0,4^{\mathrm{b}}$ & $0,52 \pm 0,10^{\mathrm{b}}$ & - \\
SG-C & $4,2 \pm 0,2^{\mathrm{a}}$ & $0,39 \pm 0,02^{\mathrm{a}}$ & $59 \pm 4^{\mathrm{a}}$ \\
SG-Cl & $4,2 \pm 0,2^{\mathrm{a}}$ & $0,43 \pm 0,02^{\mathrm{a}}$ & $56 \pm 2^{\mathrm{a}}$ \\
SG-O & $4,6 \pm 0,4^{\mathrm{a}}$ & $0,358 \pm 0,005^{\mathrm{a}}$ & $66 \pm 4^{\mathrm{b}}$
\end{tabular}

573 (1)Percentage of lost throughout film drying respect initial quantity of film forming dispersion

574 a,b, Different superscripts within the same column indicate significant differences among films $(\mathrm{p}<$ $5750.05)$. 
589 Table 3. Tensile (Elastic modulus: EM, tensile strength: TS and deformation: E at 590 break) and optical (internal transmittance: $\mathrm{Ti}$ and gloss) properties of starch-gelatin 591 based films, containing or not essential oils. C: cinnamon; Cl: clove; O: oregano. Mean 592 values, standard deviation and ANOVA results.

\begin{tabular}{|c|c|c|c|c|c|}
\hline$\overline{\text { FILMS }}$ & $\mathrm{EM}(\mathrm{MPa})$ & TS(MPa) & E (\%) & $\mathrm{Ti}$ (480nm) & Gloss $\left(60^{\circ}\right)$ \\
\hline SG & $627 \pm 54^{\mathrm{ab}}$ & $24 \pm 2^{\mathrm{ab}}$ & $14 \pm 4^{\mathrm{ab}}$ & $81 \pm 0,04^{\mathrm{a}}$ & $37 \pm 4^{\mathrm{d}}$ \\
\hline SG-C & $775 \pm 119^{b}$ & $25 \pm 2^{\mathrm{b}}$ & $8 \pm 3^{\mathrm{a}}$ & $91 \pm 0,5^{b}$ & $12 \pm 2^{a}$ \\
\hline SG-Cl & $642 \pm 71^{\mathrm{ab}}$ & $25 \pm 3^{b}$ & $17 \pm 3^{\mathrm{b}}$ & $90 \pm 0,4^{b}$ & $17 \pm 3^{b}$ \\
\hline SG-O & $612 \pm 213^{a}$ & $22 \pm 2^{\mathrm{a}}$ & $12 \pm 3^{\mathrm{ab}}$ & $92 \pm 0,4^{b}$ & $23 \pm 4^{c}$ \\
\hline
\end{tabular}

593 a,b, Different superscripts within the same column indicate significant differences among films $(\mathrm{p}<$ 594 0.05).

595

596

597

598

599

600

601

602

603

604

605

606

607 
608 Table 4. Halo (diameter) of the growth inhibition of Fusarium oxysporum (FOG) and 609 Colletotrichum Gloeosporiodes (CG) at 48 and 72 hours of incubation on plaque 610 containing discs with different films. C: cinnamon; Cl: clove; O: oregano.

\begin{tabular}{|c|c|c|c|c|}
\hline \multicolumn{5}{|c|}{ Halo of fungus growth inhibition (mm) } \\
\hline FILMS & FOG at $48 \mathrm{~h}$ & FOG at $72 \mathrm{~h}$ & CG at $48 \mathrm{~h}$ & CG at $72 \mathrm{~h}$ \\
\hline SG-C & $57 \pm 4^{\mathrm{C}}$ & $48 \pm 2^{b}$ & $49 \pm 3^{b}$ & $38 \pm 3^{c}$ \\
\hline SG-Cl & $49 \pm 2^{b}$ & $46 \pm 2^{b}$ & $52 \pm 2^{b}$ & $45 \pm 2^{b}$ \\
\hline SG-O & $42 \pm 3^{\mathrm{a}}$ & $36 \pm 2^{\mathrm{a}}$ & $41 \pm 7^{\mathrm{a}}$ & $30 \pm 7^{a}$ \\
\hline
\end{tabular}

611 a,b, Different superscripts within the same column indicate significant differences among films $(\mathrm{p}<$ $6120.05)$. 